\title{
An initial look form eastern India into the efficacy and safety of glargine $U$ 300-the new ultra long basal insulin
}

\begin{abstract}
Background: Majority of the Type 2 Diabetic (T2D) subjects in the course of the disease require insulin therapy to achieve glycaemic control. Basal insulin supported oral therapy (BOT) has become a common approach for glycaemic control nowadays thus leading to early insulin initiation The newly introduced ultra long acting basal insulin glargine u 300 is a concentrated form of the gold standard basal insulin Glargine u 100 with a different pharmacokinetic and pharmacodynamic profile $(\mathrm{PK} / \mathrm{PD})$ with claimed advantages with regards to daylong durability of action and less incidence of hypoglycaemia.
\end{abstract}

Aims and objectives: To evaluate the efficacy, and safety of Glargine U300 when used along with oral anti diabetic agents $(\mathrm{OAD})$ in insulin naive Indian T2D subjects

Materials and methods: This is a retrospective real world medical registry based observational study which looked at insulin naive patients initiated on insulin Glargine $\mathrm{U}$ 300 over and above standard of care and were followed up for a minimum of 12 weeks. DMT2 subjects presenting with osmotic symptoms or with OAD failure as per Indian Insulin Guidelines and completed the minimum 12 weeks of Glargine U 300 therapy maintaining all data and without requiring rescue doses of prandial insulin, were included. The primary efficacy parameters evaluated at the end of 12 weeks was glycated hemoglobin (HbA1c) along with of fasting plasma glucose (FPG), post prandial plasma glucose (PPPG). Safety was mainly assessed by self-reported hypoglycemia by the patients and weight gain measured at the clinic.

Results: A database of 61 patients (34 male and 27 female) was looked into and there was a statistically significant reduction of all glycaemic parameters $(p<0.001)$ and patients reached target $\mathrm{HbAlc}<7 \%$ at 12 weeks. On the safety front, average weight gain was less than a kilogram $(0.71+/-0.13 \mathrm{~kg})$ and there were 5 episodes of symptomatic hypoglycaemia and one episode of severe hypoglycaemia and no incidence of nocturnal hypoglycaemia. Severe hypoglycemia was defined as severe cognitive impairment requiring external assistance for recovery. There were no statistically significant changes in creatinine, systolic and diastolic blood pressure values though there was a statistical significant $(\mathrm{p}=0.042$ and $\mathrm{p}=0.029$ respectively) reduction in triglyceride and low density lipoprotein cholesterol.

Conclusion: Glargine U300, from this data appears to be safe and effective basal insulin for initial use in insulin naive OAD failure subjects with low risk of hypoglycaemia and insignificant weight gain.

Keywords: type 2 diabetic, basal insulin, glargine u 300, hypoglycaemia, OAD failure
Volume 8 Issue I - 2021

\author{
Soumyabrata Roy Chaudhuri,' Anirban \\ Majumder, ${ }^{2}$ Debmalya Sanyal ${ }^{2}$ \\ 'Registrar, Department of Endocrinology, KPC Medical College \\ and Hospital, Kolkata \\ ${ }^{2}$ Professor, Department of Endocrinology, KPC Medical College \\ and Hospital, Kolkata
}

Correspondence: Soumyabrata Roy Chaudhuri, MBBS, MRCP, Department of Endocrinology, KPC Medical College and Hospital , 89A Lake Town Rd, Block A, Lake Town, Kolkata-700089, India, Tel +9l 983004730I, E-mail Soumya.academics@gmail.com

Received: May 08, 2020 | Published: January 19, 2021
Abbreviations: T2D, type 2 diabetic; BOT, basal insulin supported oral therapy; PK/PD pharmacodynamic profile; OAD, oral anti diabetic agents; HbA1c, hemoglobin; FPG, fasting plasma glucose; PPPG, post prandial plasma glucose; RCT, randomised control trial

\section{Introduction}

T2D is a progressive disorder where there is continued loss of beta cell function ${ }^{1}$ with time. Oral anti-diabetic agents (OAD) are effective for initial years till at some point of time during the course of the disease, progressive loss of beta cells leads to OAD failure ${ }^{2}$ causing uncontrolled hyperglycaemia. Currently the concept of basal insulin supported oral therapy (BOT) has gained strong grounds ${ }^{3}$ wherein basal insulin is initiated and uptitrated along with other permissible OADs to achieve and maintain glycaemic control. The simplicity of basal insulin usage allows early insulinisation and paves the way for graded intensification of insulin regime later on using basal plus prandial or premixed insulin regime. ${ }^{4}$ GLA-100 formed the cornerstone of BOT therapy, however despite being regarded for long as the gold standard basal insulin, but it was not true 24 hour insulin. It also had a small peak between 6-8 hrs and the time action profile thus was not absolutely flat. ${ }^{5}$ GLA-300 arrived in recent times in the Indian scenario and claimed not only to be a concentrated form of glargine but also had a peakless true 24 hour coverage with a distinctly different $\mathrm{pK} / \mathrm{pD}$ profile than that of GLA-100. ${ }^{6}$ Thus GLA-300 came in with a promise of being a basal insulin sans the shortcomings of GLA-100. We began using GLA-300 in BOT and recorded our initial experience. We retrospectively evaluated the data to look at the efficacy and safety of GLA-300 when used in BOT in insulin naive subjects.

\section{Materials and methods}

This is a retrospective real-world registry based observational study carried out at the Endocrinology outpatient department of KPC Medical College between 1st April 2018 and 31st December 2018. 


\section{Inclusion criteria}

- Insulin naive subjects on OAD who presented with osmotic symptoms and/or conformed to the Insulin initiation criteria as per the Indian insulin initiation guidelines ${ }^{7}$ and were initiated on Glargine U 300

- Patients who were followed up for a minimum of 12 weeks

- Patients who could manage to uptitrate their insulin dose as per prespecified algorithm and maintained SMBG data

- Age between 18-70 years

\section{Exclusion criteria}

- Patients who required rescue prandial insulin

- Patients who did not titrate Glargine U300

- Patients who had important SMBG data missing referring to those patients who changed the dose without valid supporting SMBG data

- Patients who discontinued Glargine U 300 before the minimum period of 12 weeks

- Age below 18 years and above 70 year

- Pregnant, intending to be pregnant or lactating

Basal insulin was started at a dose of 0.3 units $/ \mathrm{kg}$ bodyweight at a fixed time and was uptitrated or down titrated using a prespecified titration algorithm as follows: reduce by 2 units for $<70 \mathrm{mg} / \mathrm{dL}$, no change between $70-120 \mathrm{mg} / \mathrm{dL},+2$ units for $120-140 \mathrm{mg} / \mathrm{dL},+4$ units for $140-160 \mathrm{mg} / \mathrm{dL},+6$ units for $>160 \mathrm{mg} / \mathrm{dL}$. Titration was done either via personal visit to the OPD or by telephonic assistance as per the weekly SMBG values which is the SOP (standard operating procedure) of the Endocrinology Department for basal insulin titration .The glycaemic, lipid and renal parameters along with hypoglycaemia values and number of episodes from SMBG recorded at the baseline, during the 12 week period and at the end of 12 weeks were taken up for statistical analysis.

\section{Statistical analysis}

Descriptive statistical analysis was carried out with SAS (Statistical Analysis System) version 9.2 for windows, SAS Institute Inc. Cary, NC, USA and Statistical Package for Social Sciences (SPSS Complex Samples) Version 21.0 for windows, SPSS, Inc., Chicago, IL, USA, with Microsoft Word and Excel being used to generate graphs and tables. Results on continuous measurements are presented as mean \pm $\mathrm{SD}$ and results on categorical measurements are presented in number (\%). Paired t-test was used to find the significance of study parameters between the baseline and the follow-up visits. Significance is assessed at a level of $5 \%$.

\section{Results}

Browsing through the EMR (electronic medical records) a total dataset of 61 patients was obtained after matching the inclusion and exclusion criteria. Table $1 \& 2$ amongst the lipid profile parameters LDL cholesterol $(p=0.029)$ and triglyceride $(p=0.042)$ reached statistical significance. The dosage of insulin (Glargine U 300) was increased from $19.72+/-1.62$ units to $20.95+/-1.73$ units and thus failed to attain statistical significance $(\mathrm{p}=0.655)$ as did creatinine $(\mathrm{p}=0.702)$. Hypoglycemia data was retrieved from the regularly maintained EMR system of our department from available SMBG records and telephonic inputs. There were five episodes of symptomatic documented hypoglycaemia, one episode of severe hypoglycaemia and no episode of nocturnal (occurring between 12:00 midnight an 6:00 AM) hypoglycaemia ${ }^{8}$ was recorded.

Table I Baseline characteristics of the patients, $n=6$ I

\begin{tabular}{|c|c|}
\hline Parameters & Values \\
\hline Male, n (\%) & $34(55.74)$ \\
\hline Female, n (\%) & $27(44.26)$ \\
\hline Age (years), Mean \pm SD & $56 \pm 9$ \\
\hline Body weight $(\mathrm{kg})$, Mean \pm SD & $69.9 \pm 1.94$ \\
\hline BMI $(\mathrm{kg} / \mathrm{m} 2)$, Mean \pm SD & $29.17 \pm 2.54$ \\
\hline $\mathrm{SBP}(\mathrm{mmHg})$, Mean $\pm \mathrm{SD}$ & $128.61 \pm 1.63$ \\
\hline $\mathrm{DBP}(\mathrm{mmHg})$, Mean $\pm \mathrm{SD}$ & $80.3 \pm 1.16$ \\
\hline FPG $(\mathrm{mg} / \mathrm{dL})$, Mean $\pm \mathrm{SD}$ & $215 \pm 13.4$ \\
\hline PPG (mg/dL), Mean \pm SD & $291 \pm 20.11$ \\
\hline $\mathrm{HbAlc}(\%)$, Mean $\pm \mathrm{SD}$ & $9.21 \pm 0.28$ \\
\hline Total Cholesterol, $(\mathrm{mg} / \mathrm{dL})$, Mean \pm SD & $146.19 \pm 4.89$ \\
\hline LDL-Cholesterol, $(\mathrm{mg} / \mathrm{dL})$, Mean $\pm \mathrm{SD}$ & $12 \mid \pm 4.26$ \\
\hline HDL-Cholesterol, (mg/dL), Mean \pm SD & $45.49 \pm 1.92$ \\
\hline Triglyceride, $(\mathrm{mg} / \mathrm{dL})$, Mean $\pm \mathrm{SD}$ & $166.34 \pm 10.64$ \\
\hline Serum Creatinine, $(\mathrm{mg} / \mathrm{dL})$, Mean $\pm \mathrm{SD}$ & $1 \pm 0.10$ \\
\hline Insulin Dose (IU), Mean \pm SD & $19.72 \pm 1.65$ \\
\hline
\end{tabular}

At the end of a treatment period of a minimum of 12 weeks with a mean duration of follow-up visit as $12 \pm 1.88$ weeks, there were statistically significant reductions $(p<0.00 I)$ of glycaemic parameters.

Table 2 Change in study parameters during the follow-up period

\begin{tabular}{|c|c|c|c|}
\hline & $\begin{array}{l}\text { Baseline } \\
\text { Mean } \pm \text { SD }\end{array}$ & $\begin{array}{l}\text { Follow-up } \\
\text { Mean } \pm \text { SD }\end{array}$ & $\mathbf{P}$ \\
\hline Body weight (kg) & $69.9 \pm 1.94$ & $70.61 \pm 2.07$ & 0.712 \\
\hline BMI (kg/m2) & $29.17 \pm 2.54$ & $29.49 \pm 2.58$ & 0.837 \\
\hline $\mathrm{SBP}(\mathrm{mmHg})$ & $128.6 \mid \pm 1.63$ & $127.6 \pm 1.52$ & 0.714 \\
\hline DBP (mmHg) & $80.3 \pm 1.16$ & $79 \pm 0.90$ & 0.802 \\
\hline FPG (mg/dL) & $215 \pm 13.4$ & $153 \pm 9.39$ & $<0.001$ \\
\hline PPG (mg/dL) & $291 \pm 20.11$ & $220 \pm 14.6$ & $<0.001$ \\
\hline HbAlc (\%) & $9.21 \pm 0.28$ & $8.42 \pm 0.27$ & $<0.001$ \\
\hline Total Cholesterol, (mg/dL) & $146.19 \pm 4.89$ & $160.5 \pm 8.06$ & 0.087 \\
\hline LDL-Cholesterol, (mg/dL) & $121 \pm 4.26$ & $76.6 \pm 4.47$ & 0.029 \\
\hline HDL-Cholesterol, (mg/dL) & $45.49 \pm 1.92$ & $44.3 \pm 1.77$ & 0.615 \\
\hline Triglyceride, (mg/dL) & $166.34 \pm 10.64$ & $142.2 \pm 19.14$ & 0.042 \\
\hline Serum Creatinine, $(\mathrm{mg} / \mathrm{dL})$ & $1 \pm 0.10$ & $0.85 \pm 0.05$ & 0.702 \\
\hline Insulin Dose (IU) & $19.72 \pm 1.65^{*}$ & $20.95 \pm 1.73$ & 0.655 \\
\hline
\end{tabular}

$\mathrm{p}<0.05$ considered as statistically significant, $\mathrm{p}$ computed by paired $\mathrm{t}$ test. * Average starting insulin dose 


\section{Discussion}

This study retrospectively evaluated the efficacy and safety of Glargine u300, the new ultra long acting basal insulin when used in insulin naive patients presenting with $\mathrm{OAD}$ failure. A comparator arm with GLA-100 would have been the ideal scenario but the GLA300 came with complementary glucose meter and hence the SMBG protocol could be easily followed up whereas there was a patient inertia to purchase a glucose meter and follow the SMBG protocol, hence within the stipulated period of time the numbers in the GLA-100 arm were far too few to serve the purpose. In absence of a comparator arm, we resorted to EDITION 3 data $^{8}$ which is a multicentre, randomised, open label parallel-group, two arm phases 3 trial that looked at the use of GLA-100 and GLA-300 as basal insulin in BOT in insulin naive subjects-exactly the same cohort of subjects we looked at in this retrospective registry study. GLA-300 flexibility of dosing (plus or minus 3 hours from a fixed timing) was not approved by the regulatory authorities of INDIA initially, hence a fixed time dosing of GLA-300 was used whereas in EDITION 3 GLA-300 was used as a flexible time dosing. The mean age of subjects using Glargine U300 was $56+/-9$ years in this study whereas in EDITION 3 trial was 58.2 $+/-9.9$ years. Age wise both cohorts were relatively well matched with a male preponderance of $55.74 \%$ in our cohort as opposed to $57.6 \%$ in EDITION 3. Body weight however was much higher in EDITION $3(95.1+/-23.3 \mathrm{~kg})$ at the baseline which is in stark contrast to the figures in our study $(69.9+/-1.94 \mathrm{~kg})$ noted at the initiation of therapy. EDITION 3 was a randomised control trial (RCT) programme where there was a 2 week screening phase, followed by a 6 month treatment period which was again followed by a 6 month safety extension period.

However the data of EDITION 3 programme at 6 months has been taken up to compare and contrast with our data of 12 week duration. Glargine U300 dose was adjusted weekly in both studies using a prespecified treatment algorithm. FPG in our study reduced from $215+/-13.4 \mathrm{mg} / \mathrm{dl}$ at baseline to $153+/-9.39 \mathrm{mg} / \mathrm{dl}$ a reduction of $62+/-4.01 \mathrm{mg} / \mathrm{dl}$ at the 12 week mark where as in the EDITION 3 trial FPG was reduced from $178.74+/-51.48 \mathrm{mg} / \mathrm{dl}$ to $120.06+/-38.88$, a reduction of $58.68+/-12.60 \mathrm{mg} / \mathrm{dl}$ at the 6 month mark, both figures being reasonably in the same range. $\mathrm{HbA1C}$ was also measured at the end of 12 weeks in this study which showed a reduction of $0.79+/-0.1$ $\%$ from the baseline of $9.21+/-0.28 \%$ whereas in EDITION 3 there was a reduction of $1.41+/-0.08 \%$ from a baseline of $8.49 \%$ over a period of 6 month. The greater reduction of HBA1c in the EDITION 3 trial despite a lower baseline value may be attributed to the longer duration of therapy, to the stringent monitoring of diet and exercise activity done by the trial coordinator and to the aggressive treatment regimen conventionally followed in an RCT like the EDITION programme. In our study, all glycaemic parameters viz attained statistically significant reduction at the 3 month, even with modest monitoring in the real world mark appears in lines with the EDITION 3 results at the 6 month mark. There were 6 episodes of documented symptomatic hypoglycaemia of which one was severe. None could satisfactorily report symptomatic non documented hypoglycaemia fulfilling the Whipple's triad. Considering all episodes of hypoglycaemia, we get a hypoglycaemia event rate of 0.43 events per participant /year which is much lower than that of annualized hypoglycaemia rate of 6.4 events per participant /year and also less than the 4.5 events of hypoglycaemia per patient/year as seen in the titration phase of GLA300 arm of EDITION 3 trial.

This large difference in incidence rates of hypoglycaemia may be attributed to the much smaller duration of our study (only12 weeks) and also due to the fact that GLA 300 shows a pronounced reduction of hypoglycaemia specially during the initial titration phase of 8 weeks as evidenced in the EDITION studies and the BRIGHT study. ${ }^{9}$ Another contributing factor to this spectacularly low rate of hypoglycaemia obtained in our study may be due to much lower rates of SMBG done in comparison to EDITION 3 which is an RCT. Finally, failure to elucidate the non-documented symptomatic hypoglycaemia may have also been an important contributor towards the astoundingly low rates of hypoglycaemia obtained in our study. Systolic blood pressure, diastolic blood pressure, body weight, BMI and creatinine values were followed up without any statistically significant changeover the 12 week period .As secondary findings, the study also showed statistically significant reduction of triglyceride $(p=0.042)$ which may be attributed to the better glycaemic control achieved. LDL-cholesterol values also showed statistically significant reduction $(\mathrm{p}=0.029)$ in our study which may be attributed to the concomitant optimum usage of statins as done in a real-world setting.

\section{Limitations}

Relatively small sample size of a real world evidence is a important limitation of this study .Absence of comparator arm due to low numbers of SMBG done in GLA-100 patients poses as an important limitation .At the end of the day, our study was of 12 week duration and we compared it with EDITION 3 data at 6 months which is a potential discrepancy during comparison.

\section{Conclusion}

In our experience, initiation with GLA-300 use in insulin naive subjects presenting with OAD failure was effective in reducing all glycaemic parameters at week 12 with very low incidence of overall hypoglycaemia and no incidence of nocturnal hypoglycaemia. The results obtained in this real-world study are collinear to that obtained in EDITION 3, an RCT done in a similar cohort of patients.

\section{Acknowledgments}

None.

\section{Conflicts of interest}

The authors declare that there are no conflicts of interest.

\section{Funding}

None.

\section{References}

1. Fonseca AV. Defining and characterizing the progression of type 2 diabetes. Diabetes Care. 2009;32 Suppl 2(Suppl 2 ):S151-S156.

2. Jindal S, Karla S. Developing a definition for oral antidiabetic drug (OAD) failure. J Pak Med Assoc. 2020;70(3):547-551.

3. Danne T, Bluhmki T, Seufert J, et al. Treatment intensification using long-acting insulin-predictors of future basal insulin supported oral therapy in the DIVE registry. BMC Endocr Disord. 2015;15:54.

4. Ghosh S, Unnikrishnan AG, Saboo B, et al. Evidence-based recommendations for insulin intensification strategies after basal insulin in type 2 diabetes. Diabetes Metab Syndr. 2017;11(1):S507-S521.

5. Owens DR, Matfin G, Monnier L. Basal insulin analogues in the management of diabetes mellitus: what progress have we made? Diabetes Metab Res Rev. 2014;30(2):104-119. 
6. Ghosh S, Ghosh R. Glargine-300: an updated literature review on randomized controlled trials and real-world studies. World J Diabetes. 2020;11(4):100-114.

7. Shah S, Sharma SK, Singh P, et al. Consensus evidence-based guidelines for insulin initiation, optimization and continuation in type 2 diabetes mellitus. J Assoc Physicians India. 2014;62(7 Suppl):49-54.

8. Bolli GB, Riddle MC, Bergenstal RM, et al. New insulin glargine $300 \mathrm{U}$ $\mathrm{ml}$ compared with glargine $100 \mathrm{U} / \mathrm{ml}$ in insulin-naïve people with type 2 diabetes on oral glucose-lowering drugs: a randomized controlled trial (EDITION 3). Diabetes Obes Metab. 2015;17(4):386-394.

9. Rosenstock J, Cheng A, Ritzel R, et al. More similarities than differences testing insulin glargine 300 units/mL versus insulin degludec 100 units/ $\mathrm{mL}$ in insulin-naive type 2 diabetes: the randomized head-to-head BRIGHT trial. Diabetes Care. 2018;41(10):2147-2154. 